\title{
HUMAN MEIOSIS III. ELECTRON MICROSCOPICAL ANALYSIS OF CHROMOSOME PAIRING IN AN INDIVIDUAL WITH A BALANCED TRANSLOCATION 46,XY,t(5p-;22p+)
}

\author{
by
}

\author{
PREBEN B. HOLM and SØREN W. RASMUSSEN
}

Department of Physiology, Carlsberg Laboratory

Gamle Carlsberg Vej 10, DK-2500 Copenhagen Valby

\begin{abstract}
Keywords: Reciprocal translocation, terminal deletion, human spermatocyte, recombination nodule, synaptonemal complex, three dimensional reconstruction, cri du chat syndrome
\end{abstract}

\footnotetext{
The meiotic behaviour of a balanced translocation involving the short arms of chromosomes 5 and 22 has been analyzed by reconstructions of lateral components and synaptonemal complexes at early and mid pachytene in human spermatocytes. At early pachytene, the normal chromosome 5 and the segment translocated onto chromosome 22 are paired with a synaptonemal complex in all 8 nuclei reconstructed, while pairing between the normal chromosome 22 and the translocation chromosome 5 was never observed. The pairing pattern was less regular at mid pachytene where a quadrivalent was observed in only 3 out of the 4 nuclei analyzed, while in the fourth nucleus, pairing was in part nonhomologous: the segment translocated onto chromosome 22 exhibited foldback pairing with itself and the short arm of the normal chromosome 5 was paired with the differential segment of the $\mathrm{X}$ chromosome. The short arms of translocation chromosome 5 and the normal chromosome 22 were paired in only one mid pachytene nucleus. Translocation chromosome 5 possessed in all. 4 nuclei a $200 \mathrm{~nm}$ long terminal region of condensed chromatin resembling the heterochromatin of the short arms of the acrocentric chromosomes. This together with the observation that the telomere of the short arm of translocation chromosome 5 was attached to the nuclear envelope permits the conclusion that the translocation is reciprocal with transfer of the telomere region from chromosome 22 to chromosome 5 and that of the latter to chromosome 22. Recombination nodules were more frequent in bivalent 5 than in the remainder of the genome whereas a similar increase in nodule frequency was not observed for bivalent 22 .
} 


\section{INTRODUCTION}

The nature of the terminal region of the chromatid, the telomere, and its function in the maintenance of the integrity of the uninemic chromatid is a matter of controversy. At the molecular level, speculations have concentrated on how the ends of a linear DNA molecule are replicated and in many models it has been proposed that the DNA of the telomere consists of a palindromic base sequence which is able to form a hairpin loop $(1,2,3)$. In addition, it has been suggested that the telomeres contain identical sequences which enable transient associations between different chromatids during S-phase $(3,32)$. RuBin (29) demonstrated by in situ hybridization with a cloned restriction nuclease fragment of Drosophila DNA that all telomeres of Drosophila melanogaster chromosomes shared a common 3000 base-pair unit but proposed that additional heterogeneous sequences, characteristic of individual telomeres were also present.

The hypothesis of a unipolar terminal region of the chromatid has been of special importance in the study and interpretation of chromosome breaks and rearrangements. MULLER and coworkers $(20,21)$ obtained evidence that $\mathrm{X}$-ray induced breaks in Drosophila rarely lead to stable terminal deletions and terminal inversions and proposed that the terminal parts of a chromatid consist of special chromomeres, the telomeres, which are unable to fuse with a broken chromosome segment. They arrived at the conclusion that a stable chromosome rearrangement had to be the result of a two-break event.

The concept of the telomere as a unique and highly specialized part of the chromatid has been questioned repeatedly. With the aid of salivary gland chromosome analysis, several stable and genuine terminal deletions have been claimed for Drosophila $(5,31)$ but most of these have later been reinterpreted (cf. MULLER and HERSKOWITZ, 21) and in a recent investigation, ROBERTS (28) agreed with MULLER that stable terminal deletions of the Drosophila $\mathrm{X}$ chromosome were extremely rare if present at all.

In other organisms, the evidence for and against terminal deletions is likewise incon- clusive. In a recent review on Neurospora cytogenetics, PERKINS and BARRY (24) reported that out of 167 rearrangements, 12 appeared to be nonreciprocal translocations and 3 terminal inversions. It was proposed, however, that these actually were the result of a twobreak event, but that one of the breaks was so close to the telomere that it was not detectable by genetic and light microscopical methods.

The behaviour of a true terminal deletion during mitotic divisions in maize has been analyzed in great detail by MCCLINTOCK (18). Dicentric chromosomes (which form a bridge at the first meiotic anaphase) were obtained as a result of crossing over in a maize strain heterozygous for a complex rearrangement including an inversion in chromosome 9. Upon breakage of the dicentric chromosome, different types of terminal deletions are formed. Fusion of the broken ends of the two sister chromatids leads to formation of new dicentrics which subsequently break at the following anaphase. This breakage-fusion-bridge cycle continued in the gametophyte and in the endosperm giving rise to variegation effects, whereas such effects were absent in the sporophyte. Apparently the terminally deleted chromosome had healed in the sporophyte.

In an X-ray study of Chinese hamster chromosomes, Hsu (14) proposed that healing of the ends of broken chromosomes might be attributed to the existence of "interstitial telomeres" while LiMA-DE-FARIA and SARVELLA (16) depicted polymorphisms of pachytene telomeres in Solanum and Secale which can be interpreted as partial deficiencies of the telomere region.

In man, possible genuine terminal deletions and nonreciprocal translocations have been reported several times (e.g. 4, 7, 31). In a study of 35 individuals with a 5p-karyotype (deletion of a part of the short arm of chromosome 5), NiEBUHR (22) found 27 individuals with apparently terminal deletions, 4 with interstitial deletions, and 4 with translocations. Among the latter, one was balanced and apparently nonreciprocal (case number 4). It was argued, however, as already suggested by MULLER and HERSKOWITZ (21) that the deleted chromosome may contain an intact telomere but that the telomere is too 
small to be identified in the light microscope.

In most organisms, all chromosomes become attached to the nuclear envelope during leptotene and zygotene $(10,33)$, and in human spermatocytes, free telomeres are rarely observed during late zygotene and pachytene $(12,27)$. It has also been found that a broken end of a chromosome is never attached to the nuclear envelope suggesting that only intact telomeres can bind to the inner membrane of the nuclear envelope (27). It is thus possible to assess the question of the existence of terminal deletions and nonreciprocal translocations by analyzing the behaviour of the affected chromosomes during chromosome pairing in zygotene and early pachytene. The analysis of chromosome pairing may furthermore reveal the locations of homologous regions in the affected chromosomes.

The present analysis reports on an electron microscopical analysis of the meiotic behaviour of a balanced translocation, $46, X Y, t(5-; 22 p+)$, which in the light microscope appears to be nonreciprocal (22, case number 4 ). The rationale behind the analysis was the following: 1) If the short arm of the affected chromosome 5 lacks a telomere, the arm will not attach to the nuclear envelope. 2) If the translocation is nonreciprocal, chromosome pairing should be observed only between the short arms of the normal chromosome 5 and the affected chromosome 22. 3) At mid-late pachytene, the chromatin of the telomeric regions of the short arms of all acrocentric bivalents is condensed (12). If the translocation is reciprocal, i.e., if a telomere or telomere region is transferred from the short arm of chromosome 22 to the short arm of the deleted chromosome 5, the telomere region of the latter should contain a region of condensed chromatin.

\section{MATERIALS AND METHODS}

\subsection{Material}

A testicular biopsy from a balanced translocation heterozygote was obtained through the courtesy of Dr. P. Fogh ANDERSEN, Diakonisse Stiftelsens Hospital, Copenhagen. The proband was the father of a child with typical clinical features of the cri du chat syndrome. A cytological analysis of the mitotic chromosomes has been presented previously by NIEBUHR (22, case number 4 ). In his analysis, the translocation was characterized and designated as $46, \mathrm{XY}, \mathrm{t}(5 ; 22)$ (p14;p12). A T band was not identified on the terminal region of the short arm of the affected chromosome 5 thus indicating that a telomeric region was lacking and that the translocation was nonreciprocal.

\subsection{Electron microscopy}

Immediately after removal, the testicular biopsy was placed in a $4 \%$ phosphate buffered $(0.06 \mathrm{M}, \mathrm{pH} 7.2)$ glutaraldehyde solution with a purification index of about 0.2 , cut in smaller pieces, transferred to fresh glutaraldehyde and fixed for $3 \frac{1}{2}$ hours at room temperature. After two rinses in phosphate buffer, the tissue was postfixed in $2 \%$ osmiumtetroxide in phosphate buffer at room temperature for two hours, washed twice in buffer, 4-5 times in distilled water, and left overnight in distilled water at $4^{\circ} \mathrm{C}$. The biopsy was then fixed/stained in a $2 \%$ aqueous uranyl acetate solution at $60^{\circ} \mathrm{C}$ for 3 hours. After cooling to room temperature, the tissue was dehydrated in a graded alcohol series, embedded in SPURR's low viscosity resin and polymerized for 24 hours at $70^{\circ} \mathrm{C}$.

Thick sections (about $1 \mu \mathrm{m}$ ) of the seminiferous tubules were examined in the light microscope for a preliminary identification of meiotic stages. Nuclei in the relevant stages of meiotic prophase were serially sectioned on a Reichert Om U3 ultramicrotome equipped with a diamond knife. The serial sections were picked up on single slot grids without formvar film and thereafter transferred onto grids with supporting film, using a micromanipulator for maneuvering the grids during the transfer process. Sections were stained in a $5 \%$ aqueous uranyl acetate solution for $30 \mathrm{~min}$ at $50^{\circ} \mathrm{C}$, rinsed, and poststained in lead citrate for $20 \mathrm{~min}$. The staining was carried out in an automatic staining apparatus. Sections were examined, and selected nuclei were photographed in a Siemens Elmiscope 102 at a primary magnification of 5,000 times.

\subsection{Three dimensional reconstruction}

Three dimensional reconstructions were performed in the following way: The synaptonemal 
complex segments from groups of ten sections were traced onto transparent plastic film. After having encompassed the whole nucleus, the tracings of all synaptonemal complex segments of each bivalent were redrawn on a new sheet of plastic film together with the relevant section numbers. The projected lengths were measured on a Hewlett Packard digitizer (HP 9864A) and the absolute length in $\mu \mathrm{m}$ automatically calculated with the aid of a Hewlett Packard calculator (HP 9825A) using the Pythagorean Theorem $a^{2}+b^{2}=c^{2}$ (where $a$ is the projected length, $b$ the section thickness, and $c$ the absolute length). The estimated section thickness was $0.08 \mu \mathrm{m}$. After having measured all bivalents in a nucleus, the autosomes were automatically classified by the computer according to length and centromere indices following the criteria previously described (12). Finally, an idiogram for each nucleus was automatically drawn by a HP 9872A four colour plotter.

\section{RESULTS}

\subsection{Electron microscopy}

\subsubsection{General observations}

Seven nuclei (numbers 39-41, 47-50) in early pachytene and 4 nuclei (numbers 27-30) in mid pachytene were completely reconstructed. In addition, one early pachytene nucleus (number 42) was partially reconstructed (the reconstruction could not be completed due to missing sections). The reconstructed part contained bivalents 5 and 22 and the $X Y$ bivalent.

The ultrastructure of early and mid-late pachytene nuclei as well as the morphology of the seminiferous tubules were similar to that of normal males. Detailed descriptions of the pachytene stage in normal spermatocytes have previously been presented $(12,13,27)$.

Apart from the chromosomes involved in the translocation, the present investigation did not reveal alterations in the pairing of the chromosomes and the number of aberrations was comparable to that of normal spermatocytes. In one mid pachytene nucleus (number 27) one homologue of bivalent 9 was broken and the fragment lost. The break point was in or slightly distal to the secondary constriction. In addition, four bivalents were attached to the nu- clear envelope with only one of their ends (nucleus number 27, long arms of bivalents 7 and 22 , Figure 7 ; nucleus number 29 , short arm of bivalent 6 ; nucleus number 30 , long arm of bivalent 2). The two lateral components of these bivalents were of exactly the same length and the terminal parts of the free lateral components were somewhat thicker suggesting that the telomeres were intact.

The two chromosomes which have participated in the translocation are referred to as translocation chromosomes 5 and 22 while their normal homologues are referred to as normal chromosomes 5 and 22.

\subsubsection{Classification of autosomal bivalents}

In a previous investigation (12) 14 of the 22 autosomal bivalents were identified on the basis of length and centromere index. The remaining 8 bivalents were allocated to one of the major groups. This classification was based on the data reported by HULTÉN (15) on the relative length and centromere index of diakinesis bivalents.

In the present study, the chromosomes involved in the translocation were classified as chromosomes 22 and 4 or 5 on the basis of their length and centromere indices. It was previously proposed (12) that bivalent 5 was slightly longer than bivalent 4 and had a higher centromere index. This study demonstrates that bivalents 4 and 5 cannot be distinguished by their length (Tables I and III), whereas bivalent 5 , in 9 of 11 nuclei, had a lower centromere index than bivalent 4 .

\subsubsection{Bivalent length at early pachytene}

The mean absolute length, relative length, and centromere index for the autosomal bivalents and the $X Y$ bivalent from 7 pachytene nuclei are given in Table I. A comparison with the mean lengths and centromere indices for 14 early pachytene nuclei from three normal males does not reveal significant differences (twosample t-test, $5 \%$ probability level). Only the mean length of bivalent 16 differed slightly between the two groups. The total length of the autosomal synaptonemal complexes amounted 


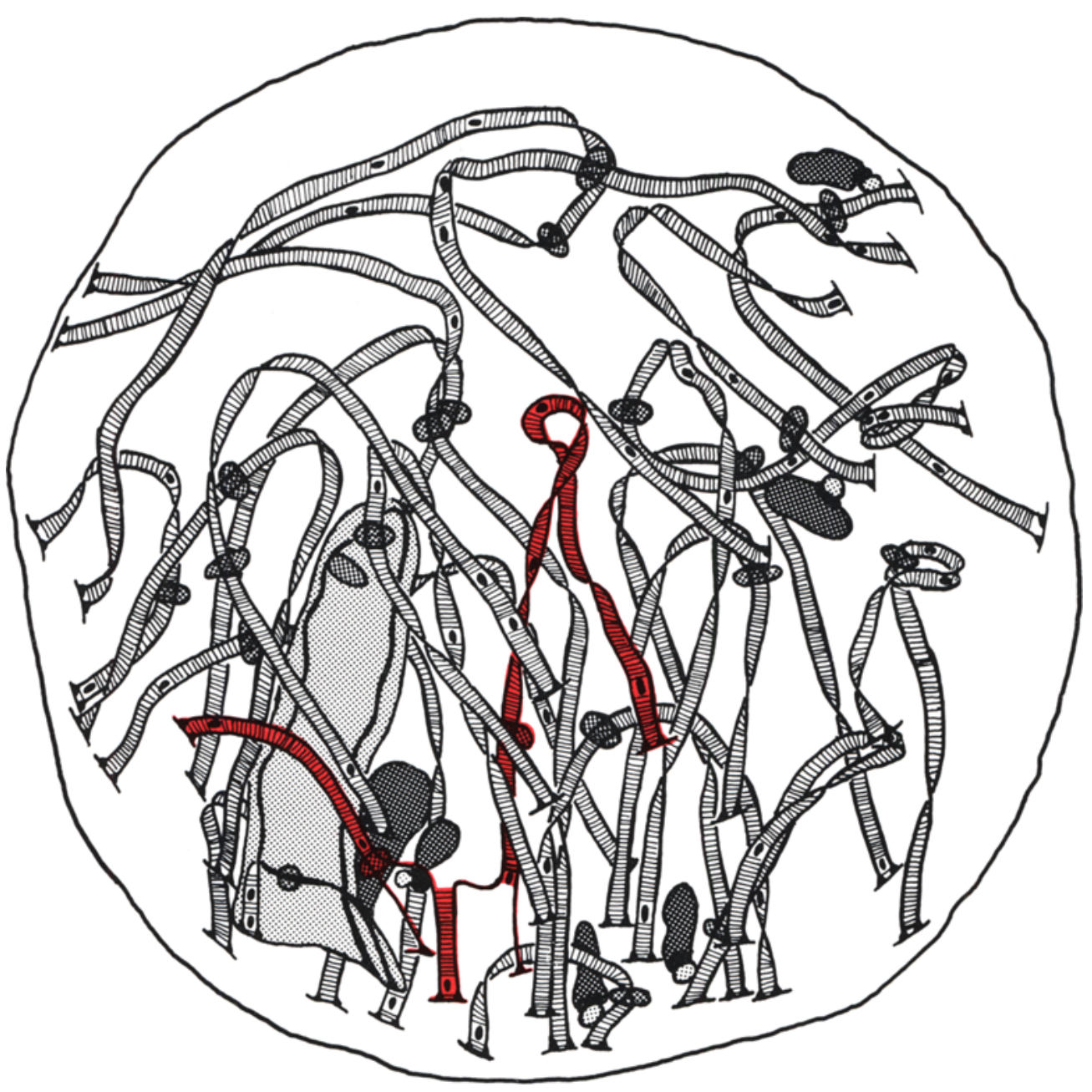

Figure 1. A complete reconstruction of the synaptonemal complexes of an early pachytene nucleus (number 49 ). The translocation quadrivalent formed by chromosomes 22 and 5 is shown in red (see also Figures 2 and 3 ). The telomeres of all bivalents are attached to the nuclear envelope and most of the attachment sites are within a limited part of the nuclear envelope (facing downwards in the reconstruction) resulting in the formation of a typical chromosome bouquet. A large number of recombination nodules can be seen in the central region of the synaptonemal complexes. The position of the centromeric heterochromatin of the bivalents is shown by an intermediate hatching. The $X Y$ bivalent (light hatching) is attached to the nuclear envelope close to the quadrivalent. The $X$ and $Y$ chromosomes are paired with a synaptonemal complex extending $0.8 \mu \mathrm{m}$ from the telomeres. Note the two recombination nodules in the paired-segment of the $X Y$ bivalent. The shorter $Y$ chromosome is attached to a nucleolus-like structure (dark hatching) and has a small heterochromatic region (intermediate hatching) which marks the position of the centromere. The lateral component of the $\mathrm{X}$ chromosome is associated with a body of similar structure. In other nuclei at this stage, up to six of these knobs are present on the $\mathrm{X}$ chromosome (27). All five acrocentric bivalents are connected with a nucleolus through a spherical body of electron transparent material (light hatching). The actual size of the nucleoli is reduced for the sake of clarity. 
Table I

Absolute length, relative length, centromere index of synaptonemal complexes, and the number of recombination nodules. The data are the mean values and standard deviations for 7 early pachytene nuclei. The data for bivalents 5 and 22 , and the $X-Y$ bivalent are from 8 nuclei.

\begin{tabular}{|c|c|c|c|c|c|c|c|c|}
\hline $\begin{array}{l}\text { Bivalent } \\
\text { number }\end{array}$ & $\begin{array}{l}\text { Absolute } \\
\text { length }(\mu \mathrm{m})\end{array}$ & $\begin{array}{l}\text { Standard } \\
\text { deviation }\end{array}$ & $\begin{array}{l}\text { Relative } \\
\text { length(\%) }\end{array}$ & $\begin{array}{l}\text { Standard } \\
\text { deviation }\end{array}$ & $\begin{array}{l}\text { Centromere } \\
\text { index }\end{array}$ & $\begin{array}{l}\text { Standard } \\
\text { deviation }\end{array}$ & $\begin{array}{l}\text { Number of } \\
\text { nodules }\end{array}$ & $\begin{array}{l}\text { Standard } \\
\text { deviation }\end{array}$ \\
\hline 1 & 17.9 & 1.6 & 8.3 & 0.3 & 49 & 6 & 7.7 & 2.8 \\
\hline 2 & 16.9 & 1.5 & 7.8 & 0.3 & 39 & 3 & 5.6 & 2.2 \\
\hline 3 & 14.2 & 2.1 & 6.5 & 0.7 & 47 & 2 & 4.0 & 1.3 \\
\hline 4 & 13.0 & 1.6 & 6.0 & 1.3 & 35 & 6 & 3.7 & 1.6 \\
\hline 5 & 13.0 & 1.6 & 5.8 & 0.4 & 29 & 2 & 5.6 & 1.9 \\
\hline 6 & 11.9 & 1.3 & 5.5 & 0.4 & 39 & 3 & 4.6 & 1.7 \\
\hline 7 & 11.5 & 1.2 & 5.3 & 0.5 & 35 & 5 & 4.9 & 2.2 \\
\hline 8 & 10.1 & 1.2 & 4.7 & 0.2 & 36 & 4 & 3.1 & 1.1 \\
\hline 9 & 10.6 & 1.5 & 4.9 & 0.5 & 38 & 5 & 4.4 & 2.3 \\
\hline 10 & 10.6 & 1.2 & 4.9 & 0.2 & 37 & 4 & 3.9 & 2.1 \\
\hline 11 & 9.6 & 1.3 & 4.4 & 0.3 & 38 & 6 & 3.6 & 1.5 \\
\hline 12 & 10.5 & 1.5 & 4.8 & 0.4 & 29 & 1 & 2.9 & 1.5 \\
\hline 13 & 8.3 & 1.1 & 3.8 & 0.2 & 13 & 4 & 3.0 & 1.0 \\
\hline 14 & 7.7 & 0.9 & 3.5 & 0.1 & 15 & 3 & 2.3 & 1.4 \\
\hline 15 & 7.2 & 0.6 & 3.4 & 0.1 & 11 & 3 & 2.9 & 1.2 \\
\hline 16 & 8.2 & 0.6 & 3.8 & 0.4 & 42 & 5 & 2.6 & 1.5 \\
\hline 17 & 8.7 & 1.1 & 4.0 & 0.3 & 33 & 2 & 3.0 & 1.5 \\
\hline 18 & 5.8 & 0.8 & 2.7 & 0.3 & 29 & 7 & 1.4 & 1.0 \\
\hline 19 & 5.5 & 0.7 & 2.6 & 0.3 & 44 & 3 & 1.4 & 0.8 \\
\hline 20 & 7.2 & 0.8 & 3.3 & 0.2 & 41 & 4 & 2.3 & 1.3 \\
\hline 21 & 3.6 & 0.5 & 1.7 & 0.1 & 24 & 5 & 1.0 & 0.6 \\
\hline 22 & 5.1 & 1.0 & 2.2 & 0.3 & 27 & 6 & 1.0 & 0.5 \\
\hline$X$ & 11.6 & 1.2 & - & - & - & - & 1.0 & 0.5 \\
\hline $\mathrm{Y}$ & 3.7 & 0.7 & - & - & 36 & 6 & & \\
\hline
\end{tabular}

to $217 \pm 21 \mu \mathrm{m}$ in the translocation heterozygote as compared to a total length of $203 \pm 23$ $\mu \mathrm{m}$ in normal males. The difference in total length as judged by a two-sample t-test was not significant at the $5 \%$ probability level.

\subsubsection{Number of recombination nodules at early pachytene}

The mean number of recombination nodules per bivalent in 7 early pachytene nuclei is given in Table I. A comparison between the number of nodules per bivalent in the translocation heterozygote and the normal material did not reveal significant differences except for bivalents 5, 9, and 18 (two-sample t-test, 5\% probability level. Only bivalent 5 showed a significant difference at the $2 \%$ level). The mean number of recombination nodules in bivalent 5 in normal spermatocytes was $3.4 \pm 2.1$ whereas $5.6 \pm 1.9$ nodules were present in the translocation heterozygote. The distribution of recombination nodules along the individual bivalents appeared to be similar in the two groups although the number of nuclei was insufficient for a reliable statistical analysis (cf. 27). The 
total number of recombination nodules per nucleus in early pachytene was $74 \pm 11$ in the translocation carrier and $75 \pm 24$ in normal spermatocytes. This difference is not significant.

It thus appears that the presence of the translocation between chromosomes 5 and 22 does not significantly change the bivalent lengths and centromere indices nor does it alter the number and distribution of recombination nodules in the remainder of the genome. These data have therefore been included in a separate report on recombination nodules in human spermatocytes (27).

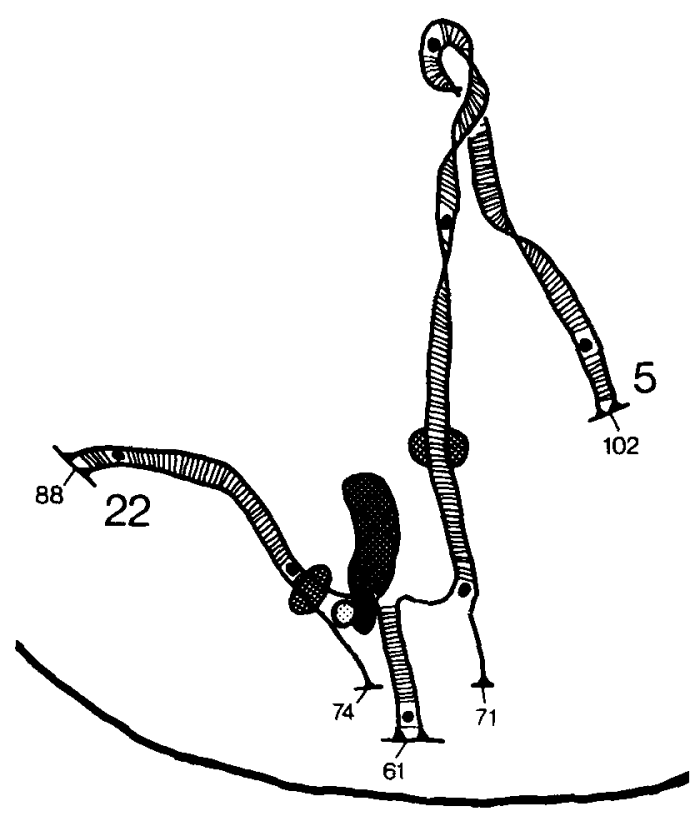

Figure 2. Reconstruction of the translocation quadrivalent formed by chromosomes 22 and 5 at early pachytene in nucleus number 49 (cf. Figures 1 and 3 ). The normal chromosome 5 is paired by a synaptonemal complex with the short arm of the translocation chromosome 22 in the region distal to the nucleolus organizer region. A recombination nodule is present in the central region of the complex close to the telomeres. The short arms of the translocation chromosome 5 and the normal chromosome 22 are both anchored at the inner membrane of the nuclear envelope. The short arm of the normal chromosome 22 is not associated with a nucleolus.

\subsubsection{Pairing of chromosomes 5 and 22 at early pachytene}

Some of the characteristics of an early pachytene nucleus are illustrated by the complete reconstruction in Figure 1. All bivalents have continuous synaptonemal complexes throughout their entire length. The telomeres of all chromosomes, including translocation chromosomes 5 and 22 and their homologues, are attached to the nuclear envelope, most of them within a limited region, giving rise to a typical chromosome bouquet.

In the 8 nuclei which were analyzed, the pairing of normal chromosomes 5 and 22 and the corresponding translocation chromosomes leads to the formation of a quadrivalent. In all nuclei pairing with synaptonemal complex formation occurred between the segment translocated onto chromosome 22 and its homologous segment on normal chromosome 5 (see Figures 2-4). None of the reconstructed quadrivalents at early pachytene revealed pairing with synaptonemal complex formation between the telomeric region of normal chromosome 22 and translocation chromosome 5 .

The association of the lateral component and the nuclear envelope is mediated through a conus of material having the same electron density as that of the lateral component. A comparison of Figures $3 f$ and $i$ shows that the terminal modification of the lateral component at its attachment site on the nuclear envelope is identical in normal chromosome 22 (Figure 3i) and translocation chromosome 5 (Figure 3 ). This demonstrates that the behaviour and morphology of the affected end of translocation chromosome 5 is indistinguishable from that of a normal telomere.

\subsubsection{Length of the translocated segment at early pachytene}

The short arm of translocation chromosome 22 possesses a continuous lateral component and is was not possible to identify the point where fusion had occurred between the short arm of chromosome 22 and the segment translocated from chromosome 5 . In all 8 pachytene nuclei, the synaptonemal complex between the 

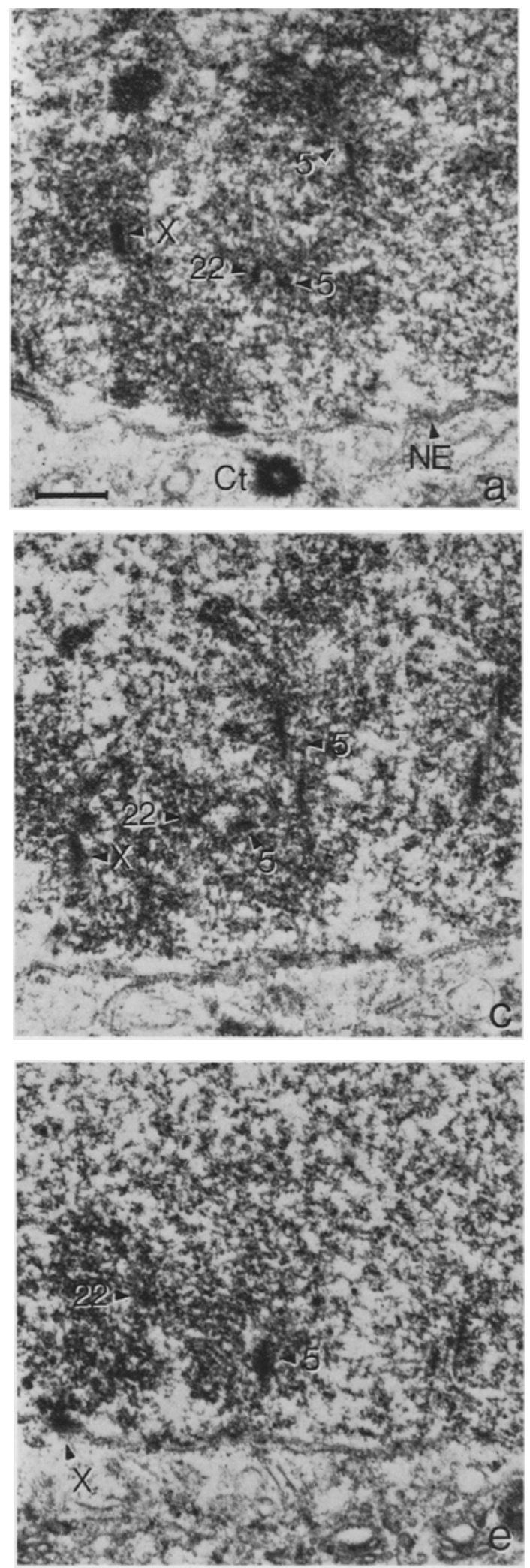
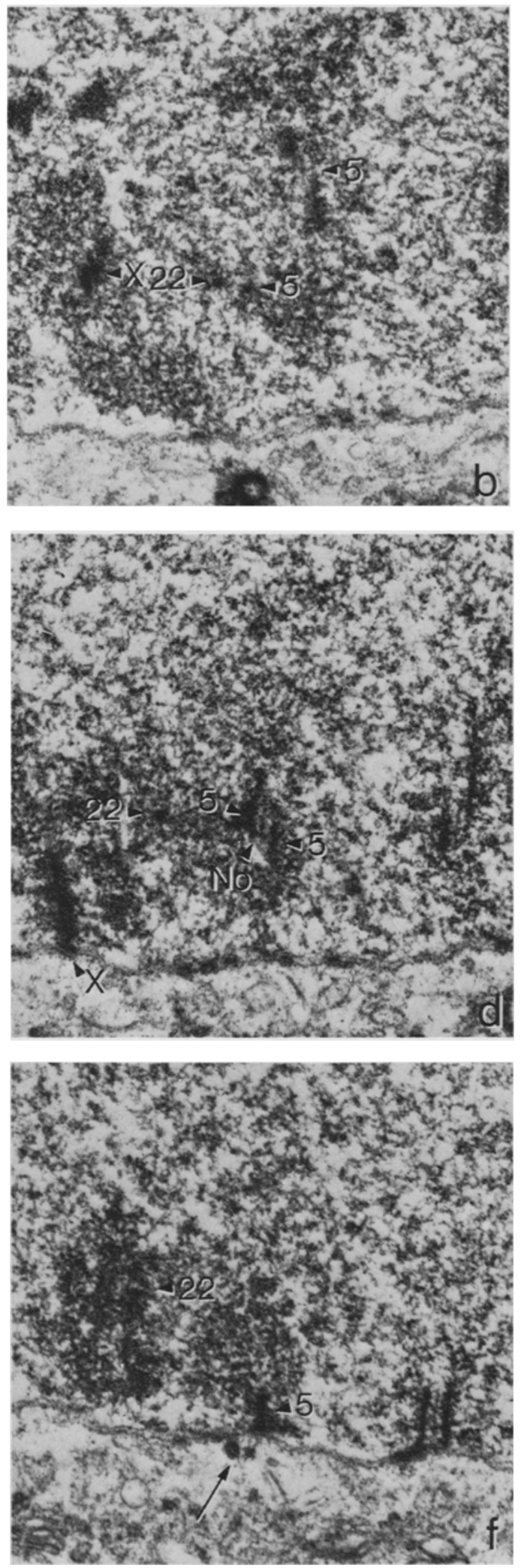

Figure 3. Electron micrographs of eleven consecutive sections and a reconstruction of a part of the quadrivalent shown in Figures 1 and 2. The arrow in Figure 3f denotes two electron dense nodes in the cytoplasm outside the attachment site of the short arm of translocation chromosome 5. 

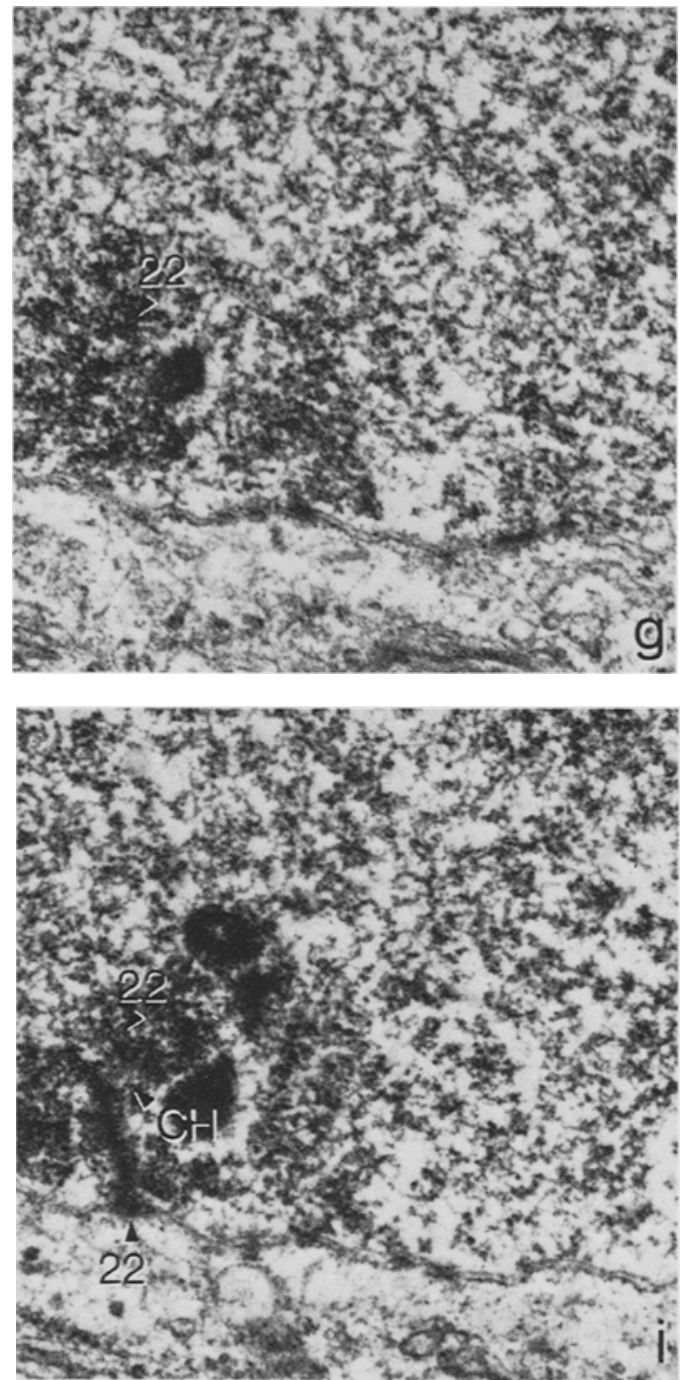
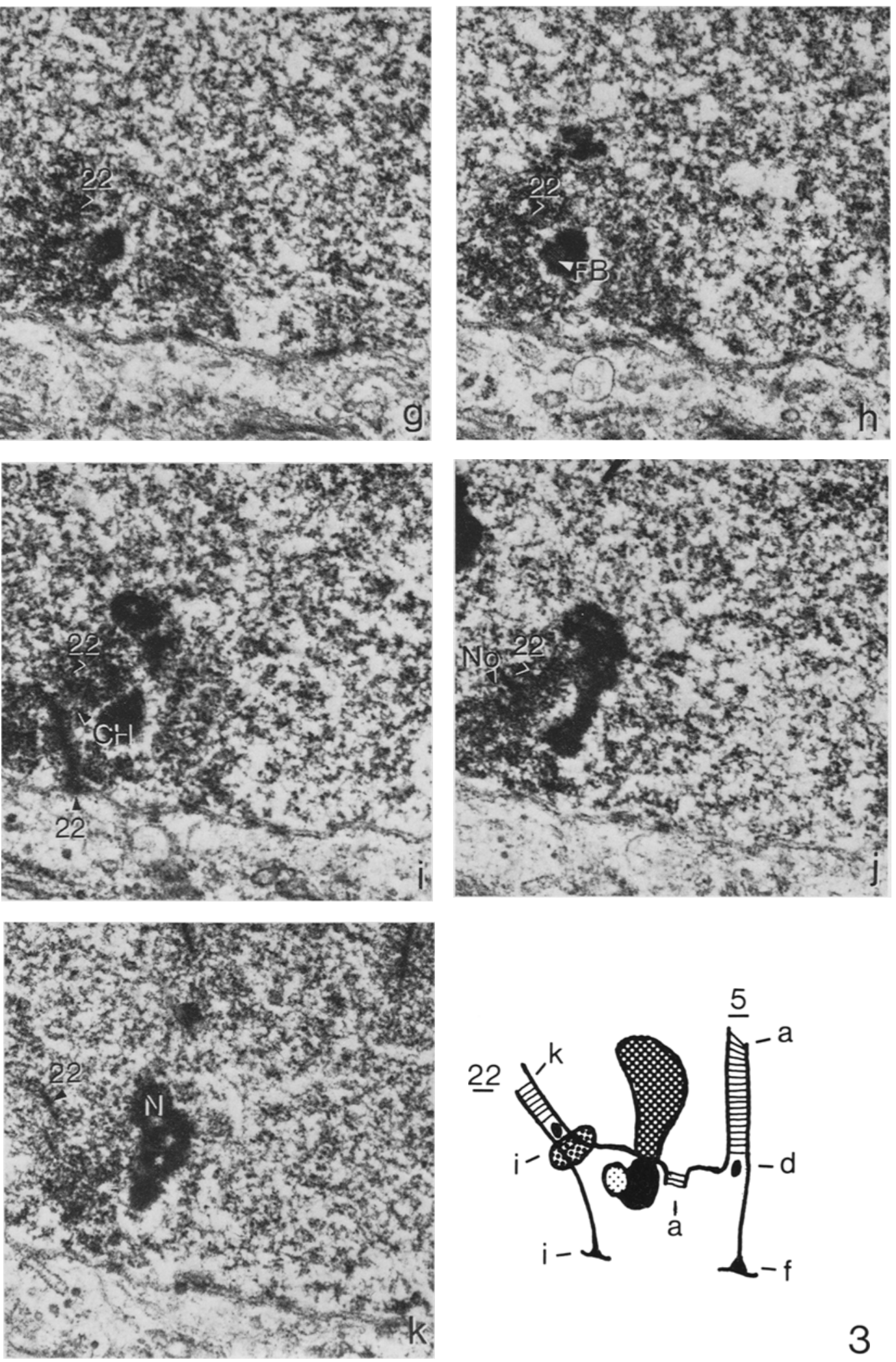

$\mathrm{X}, \mathrm{X}$ chromosome; 5 , chromosome $5 ; 22$, chromosome $22 ; \mathrm{NE}$, nuclear envelope; $\mathrm{CH}$, centromeric heterochromatin; No, recombination nodule; $\mathrm{N}$, nucleolus; FB, fibrillar body of the nucleolus organizer region; $\mathrm{Ct}$, centriole; $\mathrm{Bar}=0.5 \mu \mathrm{m}$. 


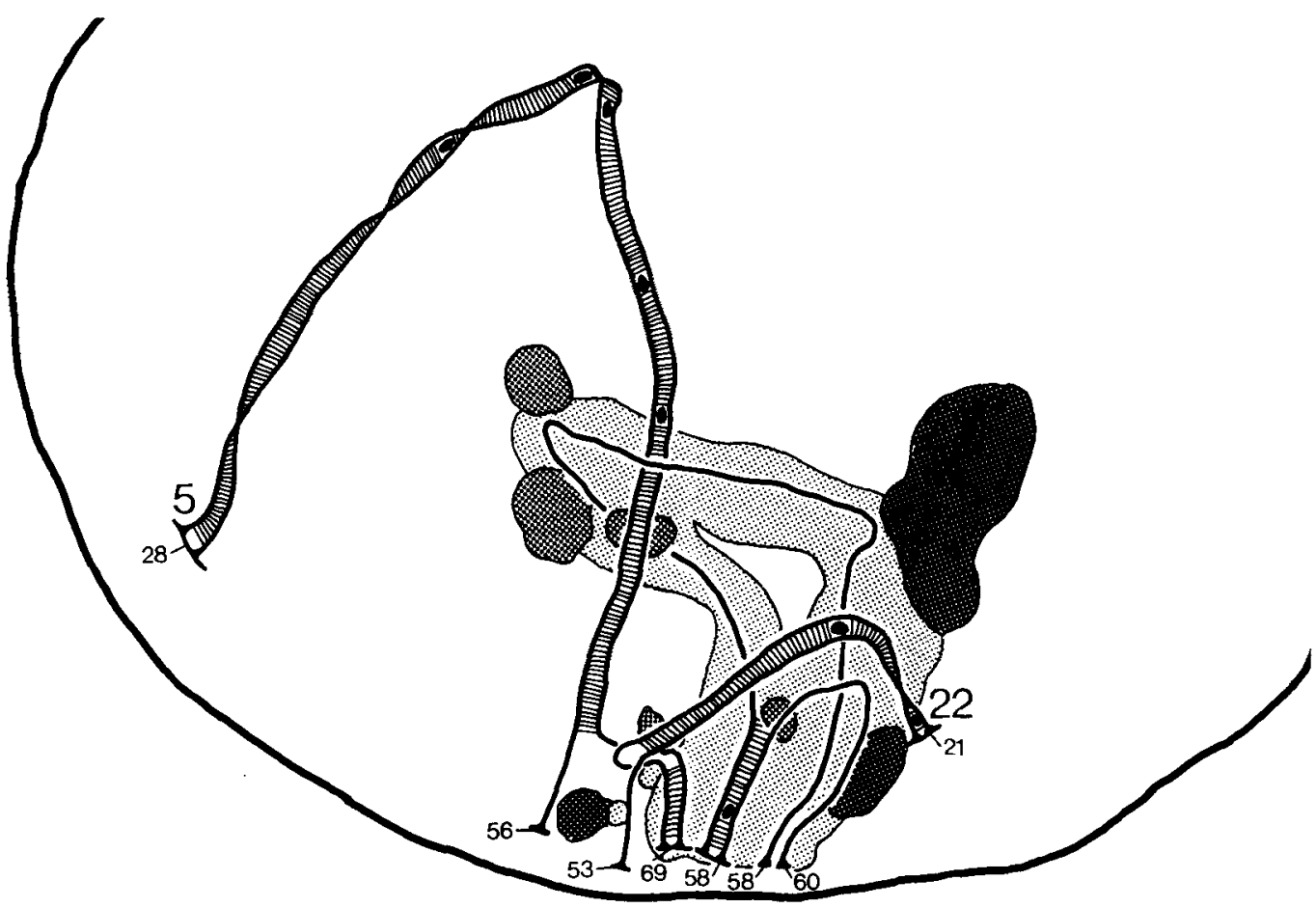

Figure 4. A reconstruction of a translocation quadrivalent and the $X Y$ bivalent at early pachytene (nucleus number 41 ). The quadrivalent is closely associated with the $X Y$ bivalent and the long $X$ chromosome forms a loop around bivalent number 5 . The synaptonemal complex between the normal chromosome 5 and the segment translocated to chromosome 22 ends a short distance from the nucleolus organizer region. Recombination nodules are not present in this stretch of synaptonemal complex. The short arm of translocation chromosome 5 is anchored at the nuclear envelope $1 \mu \mathrm{m}$ from the short arm of the normal chromosome 22 . A nucleolus is associated with this arm. The synaptonemal complex of the $X Y$ bivalent extends from the telomeres to the centromere region of the $Y$ chromosome and has one recombination nodule attached to its central region.

normal arm of chromosome 5 and translocation chromosome 22 terminates in the vicinity of the nucleolus organizer region. As illustrated in Figures 1-3, the filamentous body believed to mark the position of the ribosomal cistrons is not always in contact with the lateral component of translocation chromosome 22 . It is thus not possible to determine the distance between the nucleolus organizer and the point where the synaptonemal complex terminates.

Based on the assumption that the translocation is nonreciprocal or in case of a reciprocal translocation, that the length of the segment transferred from chromosome 22 to chromosome 5 is negligible, it is possible to estimate the length of the segment transferred from chromosome 5 to chromosome 22 in two different ways: either by measuring the length of the synaptonemal complex between the short arms of normal chromosome 5 and translocation chromosome 22 , or by measuring the difference in lateral component length between the normal chromosome and its corresponding translocation chromosome. The mean synaptonemal complex length is $1.6 \pm 0.4 \mu \mathrm{m}$ and the mean difference in lateral component length is $1.5 \pm$ $0.3 \mu \mathrm{m}$ for chromosomes 5 and $1.5 \pm 0.4 \mu \mathrm{m}$ for chromosome 22 (Table II). The mean length of the short arm of chromosome 5 is $3.8 \mu \mathrm{m}$ (Table I). The translocated segment $(1.5 \mu \mathrm{m}$, mean value) thus constitutes $39 \%$ of the length of the normal arm. This value is comparable to a value of $45-50 \%$ reported by NIEBUHR (22).

As illustrated in Table II, the differences in 


\section{Table II}

Length differences in early pachytene between the lateral components of the normal and the translocation chromosomes in bivalents 5 and 22 . The table also gives the length of the synaptonemal complex between the short arms of the normal chromosome 5 and the translocation chromosome 22 and the number of recombination nodules in this segment.

\begin{tabular}{lcccc}
\hline $\begin{array}{l}\text { Nucleus } \\
\text { number. }\end{array}$ & $\begin{array}{c}\text { Length differences between the normal } \\
\text { and the translocation chromosome }(\mu \mathrm{m}) .\end{array}$ & $\begin{array}{c}\text { Synaptonemal } \\
\text { complex length } \\
(\mu \mathrm{m}) .\end{array}$ & $\begin{array}{c}\text { Number of } \\
\text { recombination } \\
\text { nodules. }\end{array}$ \\
\cline { 2 - 3 } Bivalent 5 & Bivalent 22 & 2.4 & 1.4 & 0 \\
\hline 39 & 1.8 & 1.0 & 1.0 & 2 \\
40 & 1.0 & 1.3 & 2.0 & 0 \\
41 & 1.7 & 1.2 & 1.4 & 1 \\
42 & 1.8 & 1.5 & 1.1 & 1 \\
47 & 1.1 & 1.5 & 2.0 & 1 \\
49 & 1.2 & 1.4 & 1.5 & 0 \\
50 & 1.3 & 1.3 & 2.2 & 0.8 \\
\hline Mean & 1.8 & 1.5 & 1.6 & 0.7 \\
\hline Standard deviation & 1.5 & 0.4 & 0.4 & 1 \\
\hline
\end{tabular}

length between the normal chromosomes and the translocation chromosomes are not always identical in different nuclei. The length differences vary form 1.0 to $1.8 \mu \mathrm{m}$ for chromosome 5 and from 1.0 to $2.4 \mu \mathrm{m}$ for chromosome 22 . Furthermore, the length differences for chromosomes 5 are not always equal to those for chromosomes 22 when values from the same nucleus are compared.

\subsubsection{Recombination nodules in chromosomes 5 and 22}

The number of recombination nodules associated with the synaptonemal complex between the short arms of normal chromosome 5 and translocation chromosome 22 are presented in Table II. The mean number of recombination nodules present in this stretch of synaptonemal complex is 0.8 (corresponding to $2 \mu \mathrm{m}$ synaptonemal complex per recombination nodule). Three quadrivalents are completely devoid of nodules in this segment.

A value of $2.0 \mu \mathrm{m}$ synaptonemal complex per nodule in the affected segment of chromosome 5 is slightly lower than the mean value of 3.0 for the whole genome (mean of 21 early pachytene nuclei, 27), but cannot alone account for the significant increase in the total number of nodules on bivalent 5 of the translocation heterozygote. It thus appears that the presence of the translocation in chromosome 5 not only leads to an increase in the number of recombination nodules in the affected segment but also in the remainder of the bivalent. This effect of the translocation is not observed in bivalent 22 where a smaller but nonsignificant decrease is found.

\subsubsection{Bivalent length at mid pachytene}

The mean values for the absolute lengths, relative lengths, and centromere indices for the autosomal bivalents from 4 mid pachytene nuclei are given in Table III. A comparison between these data and the bivalent lengths and centromere indices previously published (12) does not reveal large differences. Due to the low number of mid pachytene nuclei reconstructed in the present study, a statistical analysis was not performed. The mean total length of the autosomal synaptonemal complex complement is $245 \pm 4 \mu \mathrm{m}$ in the translocation carrier and $232 \pm 19 \mu \mathrm{m}$ in normal males. 
Table III

Absolute length, relative length, and centromere index. The data are the mean values and standard deviations for 4 mid pachytene nuclei.

\begin{tabular}{|c|c|c|c|c|c|c|}
\hline $\begin{array}{l}\text { Bivalent } \\
\text { number }\end{array}$ & $\begin{array}{l}\text { Absolute } \\
\text { length }(\mu \mathrm{m})\end{array}$ & $\begin{array}{l}\text { Standard } \\
\text { deviation }\end{array}$ & $\begin{array}{l}\text { Relative } \\
\text { length(\%) }\end{array}$ & $\begin{array}{l}\text { Standard } \\
\text { deviation }\end{array}$ & $\begin{array}{l}\text { Centromere } \\
\text { index }\end{array}$ & $\begin{array}{l}\text { Standard } \\
\text { deviation }\end{array}$ \\
\hline 1 & 22.0 & 0.6 & 9.0 & 0.2 & 44 & 5 \\
\hline 2 & 20.1 & 1.0 & 8.2 & 0.4 & 40 & 4 \\
\hline 3 & 16.0 & 0.5 & 6.5 & 0.2 & 48 & 3 \\
\hline 4 & 13.8 & 0.5 & 5.7 & 0.3 & 33 & 8 \\
\hline 5 & 13.7 & 0.7 & 5.6 & 0.4 & 24 & 4 \\
\hline 6 & 13.6 & 0.7 & 5.6 & 0.2 & 40 & 2 \\
\hline 7 & 13.2 & 0.6 & 5.4 & 0.2 & 34 & 6 \\
\hline 8 & 11.4 & 1.2 & 4.6 & 0.4 & 36 & 5 \\
\hline 9 & 11.8 & 0.7 & 4.8 & 0.4 & 30 & 1 \\
\hline 10 & 11.8 & 0.5 & 4.8 & 0.3 & 40 & 3 \\
\hline 11 & 10.8 & 0.6 & 4.4 & 0.2 & 36 & 3 \\
\hline 12 & 11.4 & 0.2 & 4.7 & 0.1 & 31 & 3 \\
\hline 13 & 9.3 & 0.2 & 3.8 & 0.2 & & \\
\hline 14 & 8.5 & 0.7 & 3.5 & 0.3 & & \\
\hline 15 & 7.6 & 0.7 & 3.1 & 0.2 & & \\
\hline 16 & 9.0 & 0.5 & 3.7 & 0.2 & 47 & 1 \\
\hline 17 & 10.2 & 0.7 & 4.1 & 0.3 & 31 & 6 \\
\hline 18 & 6.3 & 0.4 & 2.6 & 0.2 & 31 & 5 \\
\hline 19 & 6.4 & 0.5 & 2.6 & 0.3 & 42 & 4 \\
\hline 20 & 8.7 & 0.3 & 3.6 & 0.1 & 43 & 1 \\
\hline 21 & 3.5 & 0.3 & 1.4 & 0.2 & & \\
\hline 22 & 6.0 & 0.4 & 2.5 & 0.2 & & \\
\hline
\end{tabular}

Very few recombination nodules were observed at this stage either in normal males or in the translocation heterozygote.

\subsubsection{Pairing of chromosomes 5 and 22}

A reconstruction of a quadrivalent consisting of chromosomes 5 and 22 is shown in Figure 5. As in early pachytene, a synaptonemal complex is present between the short arms of the normal chromosome 5 and translocation chromosome 22. The paired region as well as the unpaired regions of chromosomes 5 and 22 are surrounded by the chromatin of the XY bivalent (Figure 6). The unpaired lateral components of the quadrivalent frequently assume the same morphological characteristics as those of the $X$ and $Y$ chromosomes (regions of double lateral components, minor discontinuities, condensations, and small branched regions). All telomeres of the chromosomes constituting the quadrivalents are attached to the nuclear envelope as shown in Figures 5 and 6 . The telomere of the short arm of translocation chromosome 5 is in all four nuclei surrounded by a sphere of condensed chromatin which extends $200 \mathrm{~nm}$ from the nuclear envelope into the nucleoplasm (Figures 5, 6a, and 10). Similar, but larger regions of condensed chromatin are present in the telomeric region of the short arms of all 
acrocentric chromosomes and are confluent with the centromeric heterochromatin of these chromosomes.

Reconstructions of chromosomes 5 and 22 from the other 3 mid pachytene nuclei are shown in Figures 7-9. A diagrammatic representation of the pairing of these chromosomes is shown in Figure 10. In all these nuclei, the short arms of chromosomes 5 and 22 are intermingled with the $X Y$ bivalent. Figure 7 shows another example of the type of pairing illustrated in Figures 5 and 6 . The only example

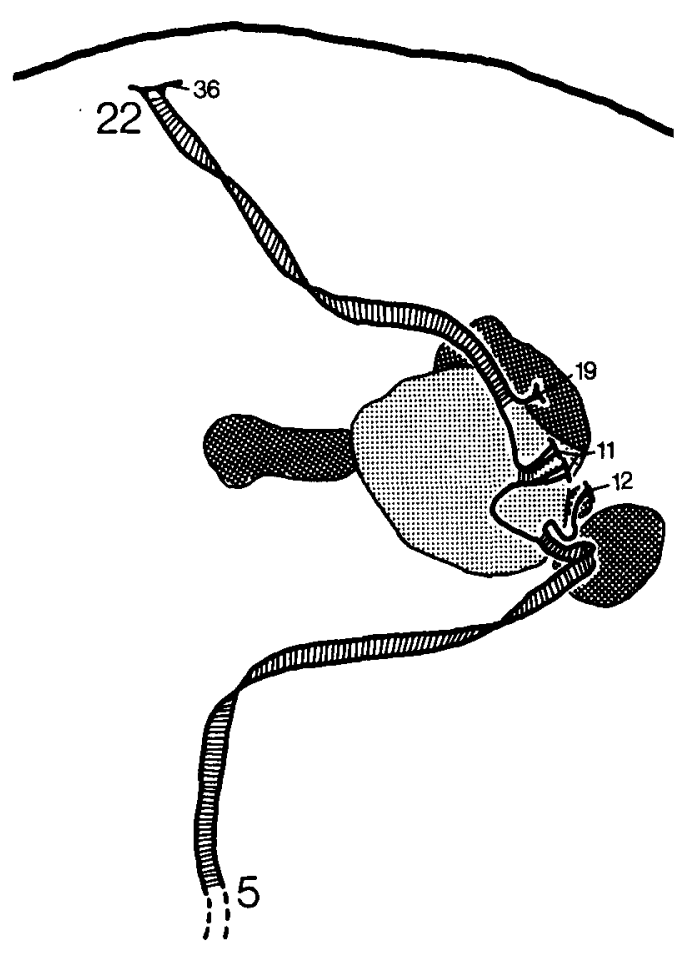

Figure 5. A reconstruction of a transiocation quadrivalent at mid pachytene (nucleus number 30 ). The quadrivalent is closely associated with the $\mathrm{XY}$ bivalent (light hatching). As in early pachytene synaptonemal complex formation is between the short arm of the normal chromosome 5 and the segment translocated onto the short arm of chromosome 22. The lateral components are double in the paired region close to the telomere and the central region of the complex appears indistinct. Note that the short arm of translocation chromosome 5 has a small region of condensed chromatin at the telomere region (see also Figure 6). of pairing with a synaptonemal complex between the short arms of the normal chromosome 22 and translocation chromosome 5 is shown in Figure 8. In one nucleus, the pairing of chromosomes 5 and 22 has not led to formation of a quadrivalent. Instead, the segment of chromosome 5 translocated onto chromosome 22 is nonhomologously paired with itself forming a hairpin structure and the short arm of normal chromosome 5 is nonhomologously paired with the long arm of the $X$ chromosome over a small distance. Outside this region, the lateral component of the $\mathrm{X}$ chromosome is double (Figure 9).

\subsubsection{Length of the translocated segment at mid pachytene}

The mean length of the synaptonemal complex between the short arms of translocation chromosome 22 and normal chromosome 5 is $1.3 \pm 0.5 \mu \mathrm{m}$ compared to $1.6 \pm 0.4 \mu \mathrm{m}$ at early pachytene. The mean differences between normal and translocation chromosomes 5 and 22 are 1.3. $\pm 0.4 \mu \mathrm{m}$ and $1.7 \pm 0.6 \mu \mathrm{m}$, respectively (Table IV). The apparent length of the translocated segment is thus similar in early and mid pachytene. As in early pachytene, there is a considerable variation in length differences between normal and translocation chromosomes when the differences for chromosomes 5 and 22 are measured in the same nucleus, the maximum difference being $1.5 \mu \mathrm{m}$ in nucleus 29 .

\section{DISCUSSION}

\subsection{The nature of the translocation}

The present study has described two observations which both strongly indicate that the short arm of translocation chromosome 5 possesses an intact and functionally active telomere. 1) In all 12 nuclei which were reconstructed, the lateral component end of this chromosome was attached to the nuclear envelope and was morphologically indistinguishable from the telomeres of the normal chromosomes. 2) The end of translocation chromosome 5 consisted in all 4 mid pachytene nuclei of a region of condensed chromatin as would be expected if its terminal segment originated 

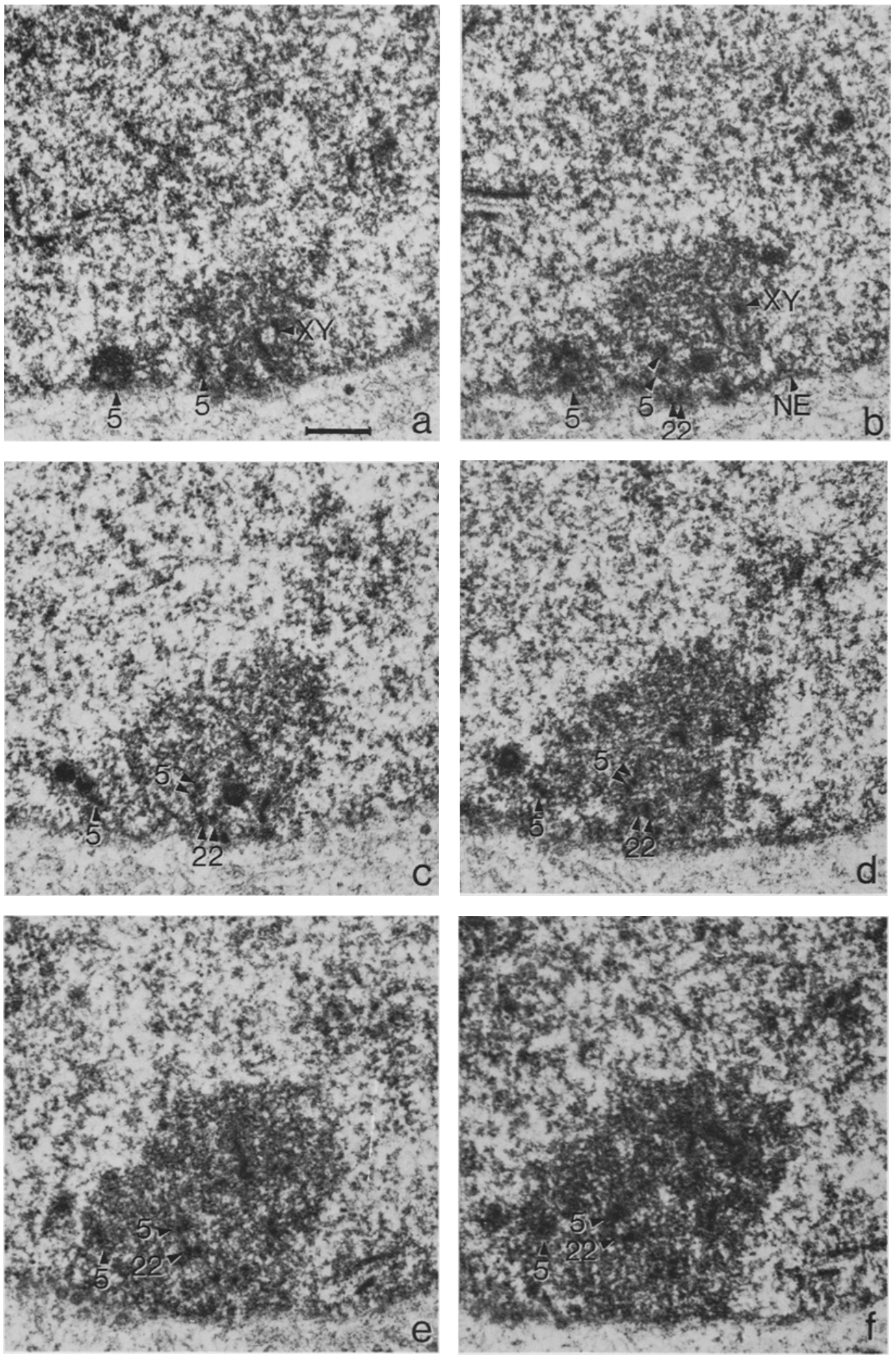

Figure 6. Electron micrographs of eleven consecutive sections and a partial reconstruction of a translocation quadrivalent at mid pachytene (nucleus number 30 ) (see also Figure 5). The part of the quadrivalent which includes the unpaired and paired regions of translocation chromosome 22 and the normal chromosome 5 is embedded in the condensed chromatin of the XY bivalent. The synaptonemal complex between chromosomes 5 and 22 

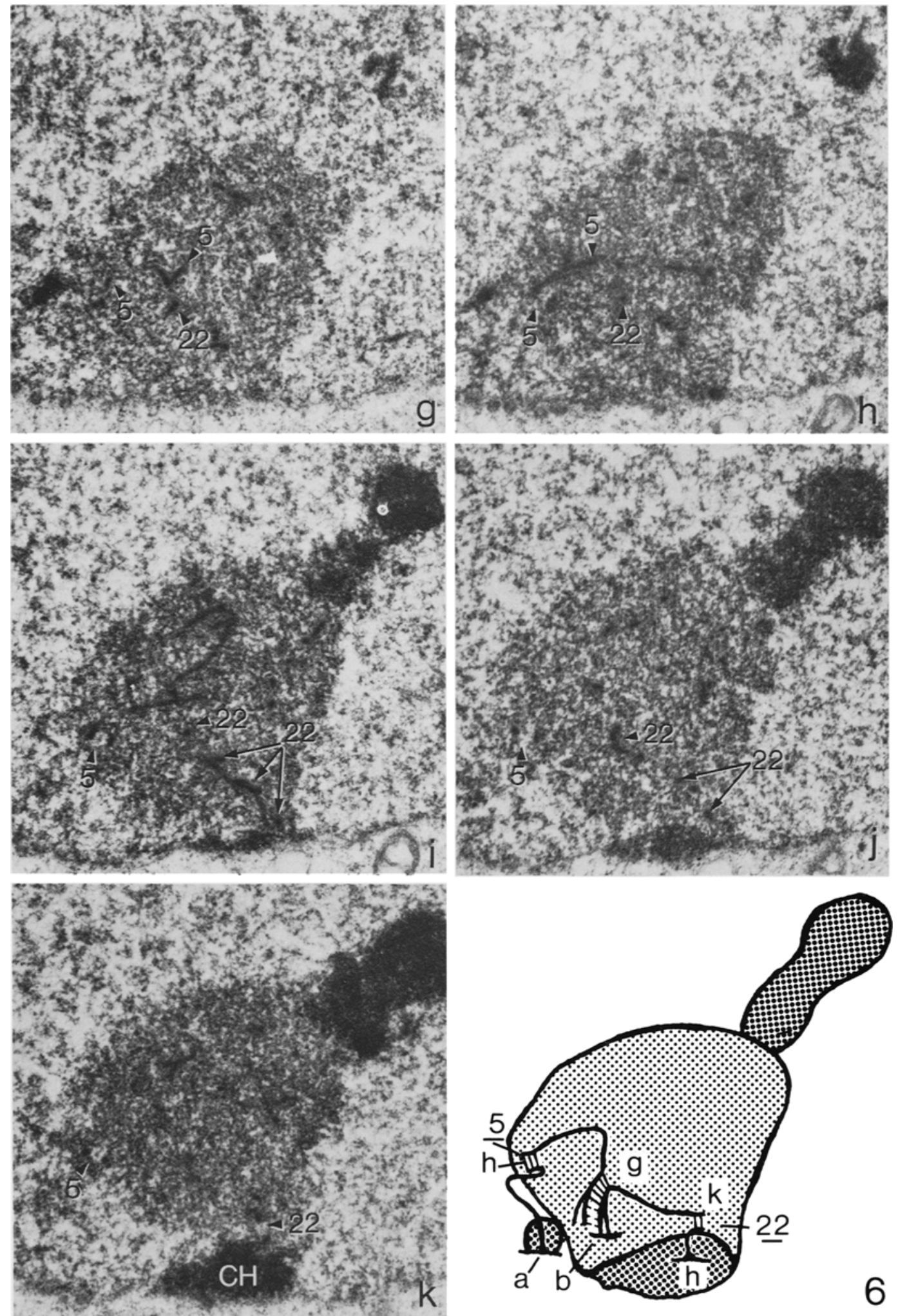

is altered in the telomere region revealing double lateral components and an indistinct central region (Figures 6b-d). The telomere region of the short arm of translocation chromosome 5 is associated with a condensed chromatin region (Figure $6 \mathrm{a}$ ). Only the paired region of the $\mathrm{XY}$ bivalent can be reconstructed at this stage (Figures 6a and b). XY, XY bivalent with synaptonemal complex; 5, chromosome 5; 22, chromosome 22; $\mathrm{NE}$, nuclear envelope; $\mathrm{CH}$, centromeric heterochromatin; $(\mathrm{Bar}=0.5 \mu \mathrm{m})$. 


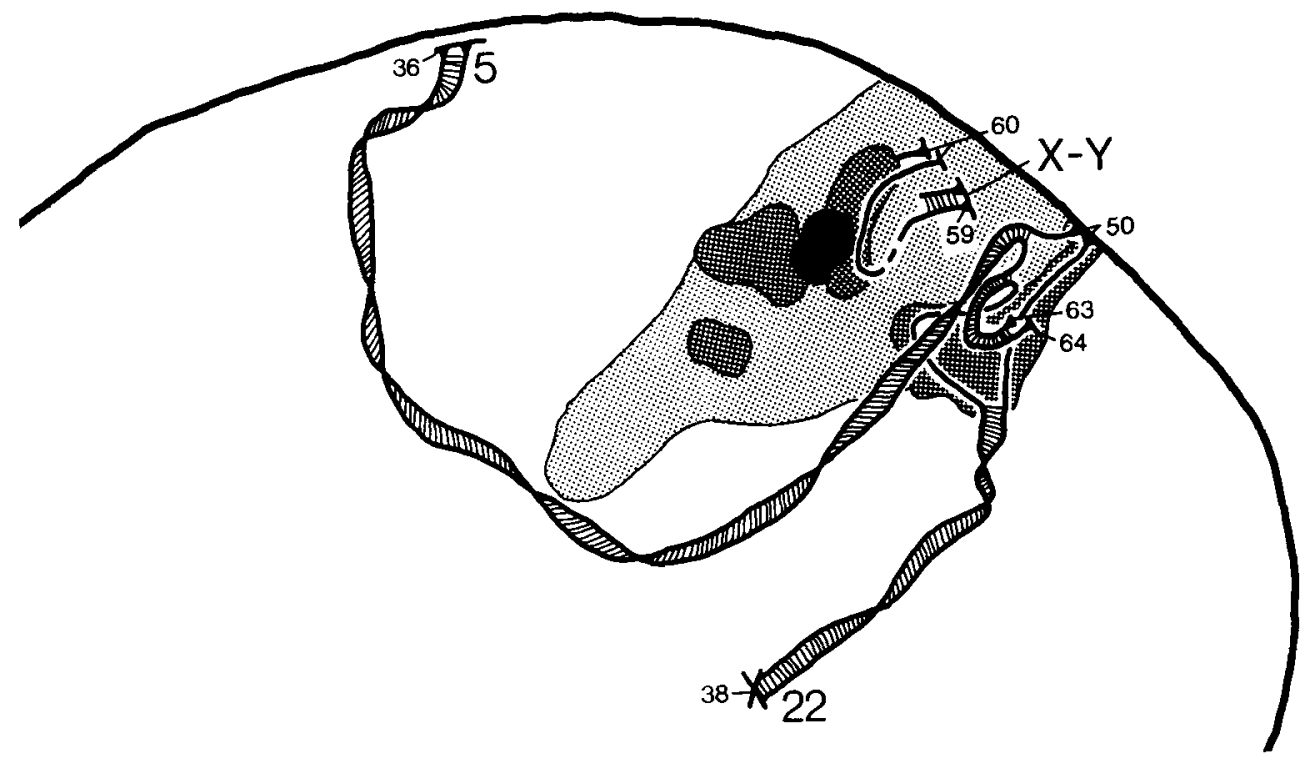

Figure 7. A reconstruction of a translocation quadrivalent in mid pachytene (nucleus number 27). The type of pairing and the association of the quadrivalent with the $\mathrm{XY}$ bivalent is similar to the quadrivalent shown in Figure 6. The short arm of translocation chromosome 5 has a small heterochromatic region at the telomere and is attached $100-150 \mathrm{~nm}$ from the lateral component of the normal chromosome 22 . The telomeres of the long arm of bjvalent 22 are free in the nucleoplasm.

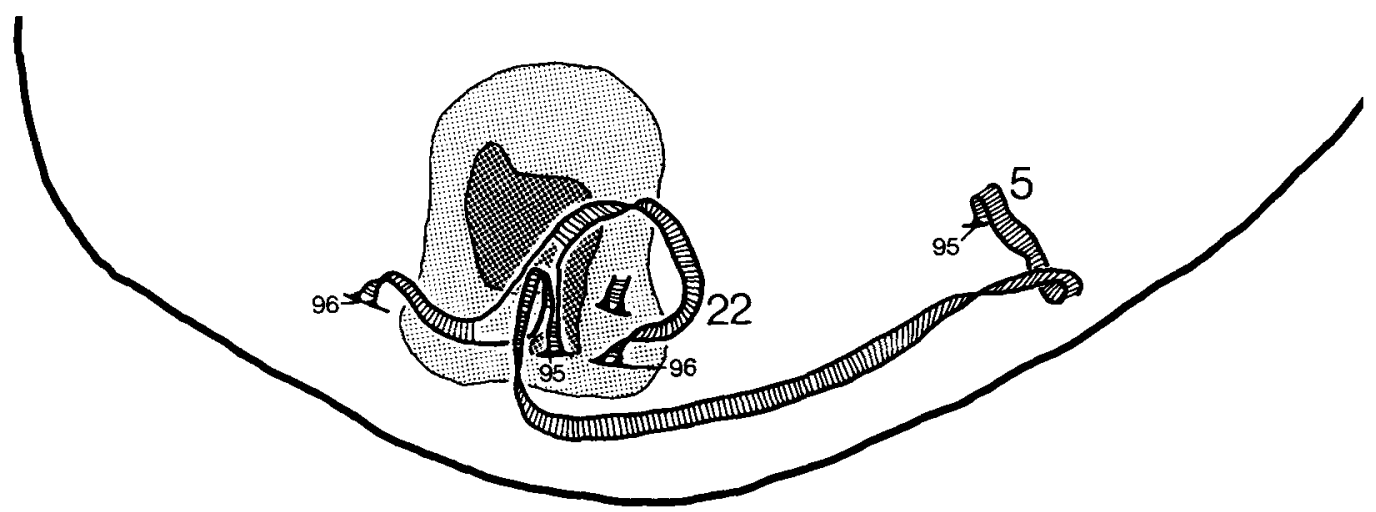

Figure 8. A reconstruction of a translocation quadrivalent at mid pachytene (nucleus number 28). The short arms of the quadrivalent are embedded in the condensed chromatin of the XY bivalent. Note that in this quadrivalent the short arms of the normal chromosome 22 and translocation chromosome 5 are also paired with a synaptonemal complex.

from the tip of the short arm of the acrocentric chromosome 22. It is therefore concluded that the translocation is reciprocal. The larger piece of chromosome 5 has been joined at a subterminal break with chromosome 22 and the 200 $\mathrm{nm}$ fragment containing one telomere of chro- mosome 22 has fused to the broken end of chromosome 5 (cf. Figure 11).

MULLER and HeRSKOWITZ (21) pointed out that the size of a functional telomere may be below the resolution of the light microscope. The present translocation supports this notion. 

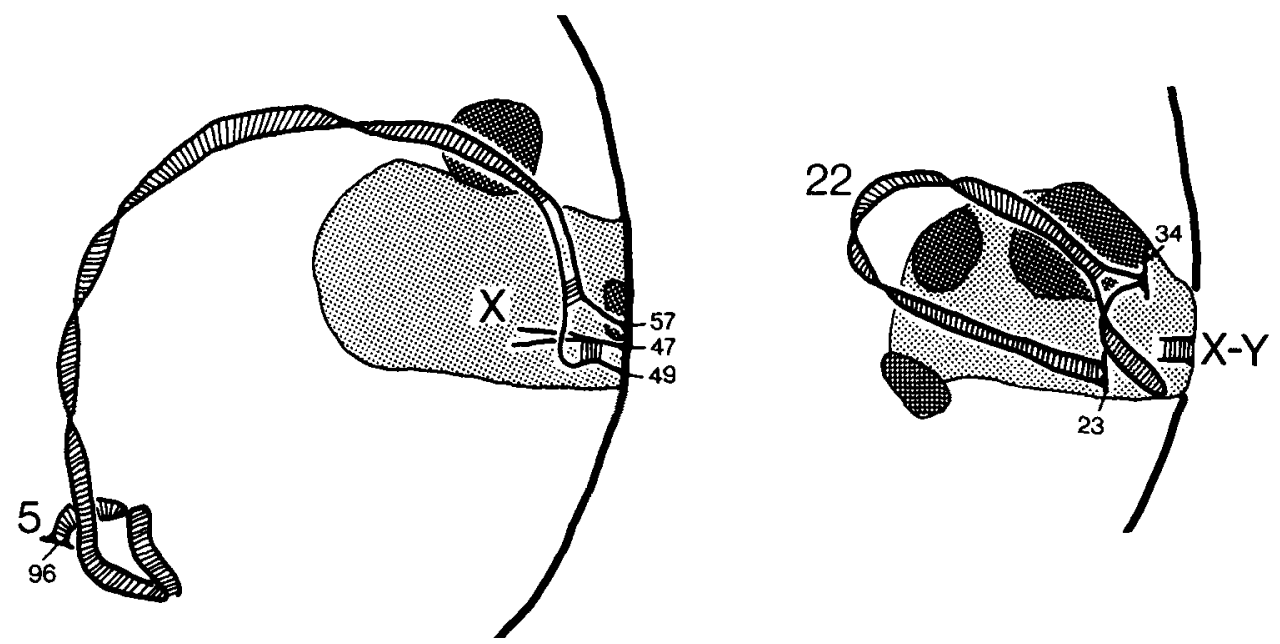

Figure 9. Reconstructions of bivalents 5 and 22 in a mid pachytene nucleus (nucleus number 29). A quadrivalent is not present in the nucleus. The short arm of translocation chromosome 22 is nonhomologously paired with itself forming a hairpin structure. A segment of the short arm of the normal chromosome 5 is nonhomologously paired by a short stretch of synaptonemal complex with the long arm of the $\mathrm{X}$ chromosome.

\section{5} 22

NUCLEUS 27 5 目县
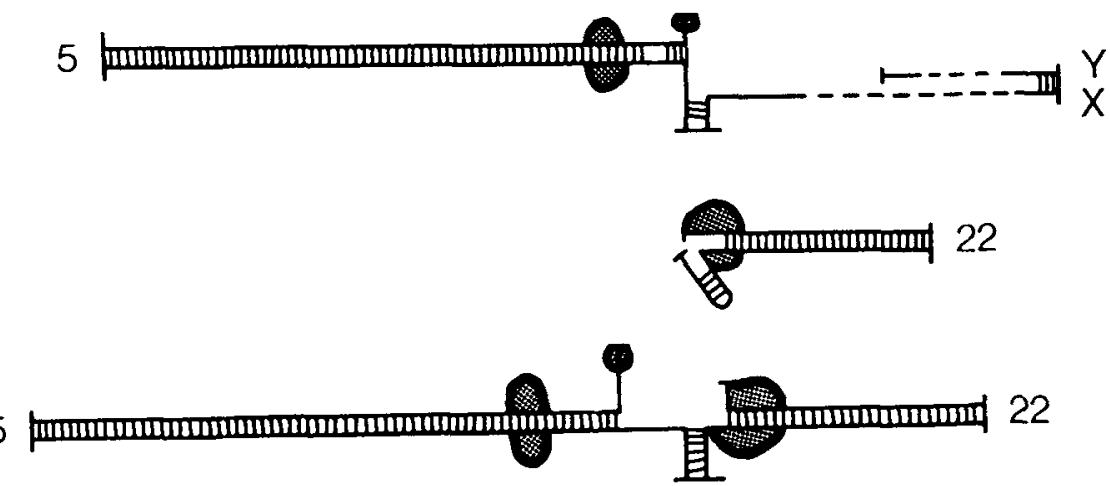

Figure 10. Schematic presentation of the pairing behaviour of chromosomes 5 and 22 at mid pachytene. Note that in all nuclei the terminal region of the short arm of translocation chromosome 5 carries a region of condensed chromation. 
Table IV

Length differences in mid pachytene between the normal and the translocation chromosomes in bivalents 5 and 22 . The table also gives the length of the synaptonemal complex between the short arms of the normal chromosome 5 and the translocation chromosome 22.

\begin{tabular}{lccc}
\hline $\begin{array}{l}\text { Nucleus } \\
\text { number }\end{array}$ & $\begin{array}{c}\text { Length differences between the normal and the } \\
\text { translocation chromosomes }(\mu \mathrm{m}) .\end{array}$ & $\begin{array}{c}\text { Synaptonemal } \\
\text { complex length }(\mu \mathrm{m}) .\end{array}$ \\
\cline { 2 - 3 } & Bivalent 5 & Bivalent 22 & \\
\hline 27 & 1.4 & 1.8 & 1.3 \\
28 & 1.8 & 1.2 & 1.7 \\
29 & 0.9 & 2.4 & - \\
30 & 1.1 & 1.2 & 0.8 \\
\hline Mean & 1.3 & 1.7 & 1.3 \\
\hline Standard deviation & 0.4 & 0.6 & 0.5 \\
\hline
\end{tabular}

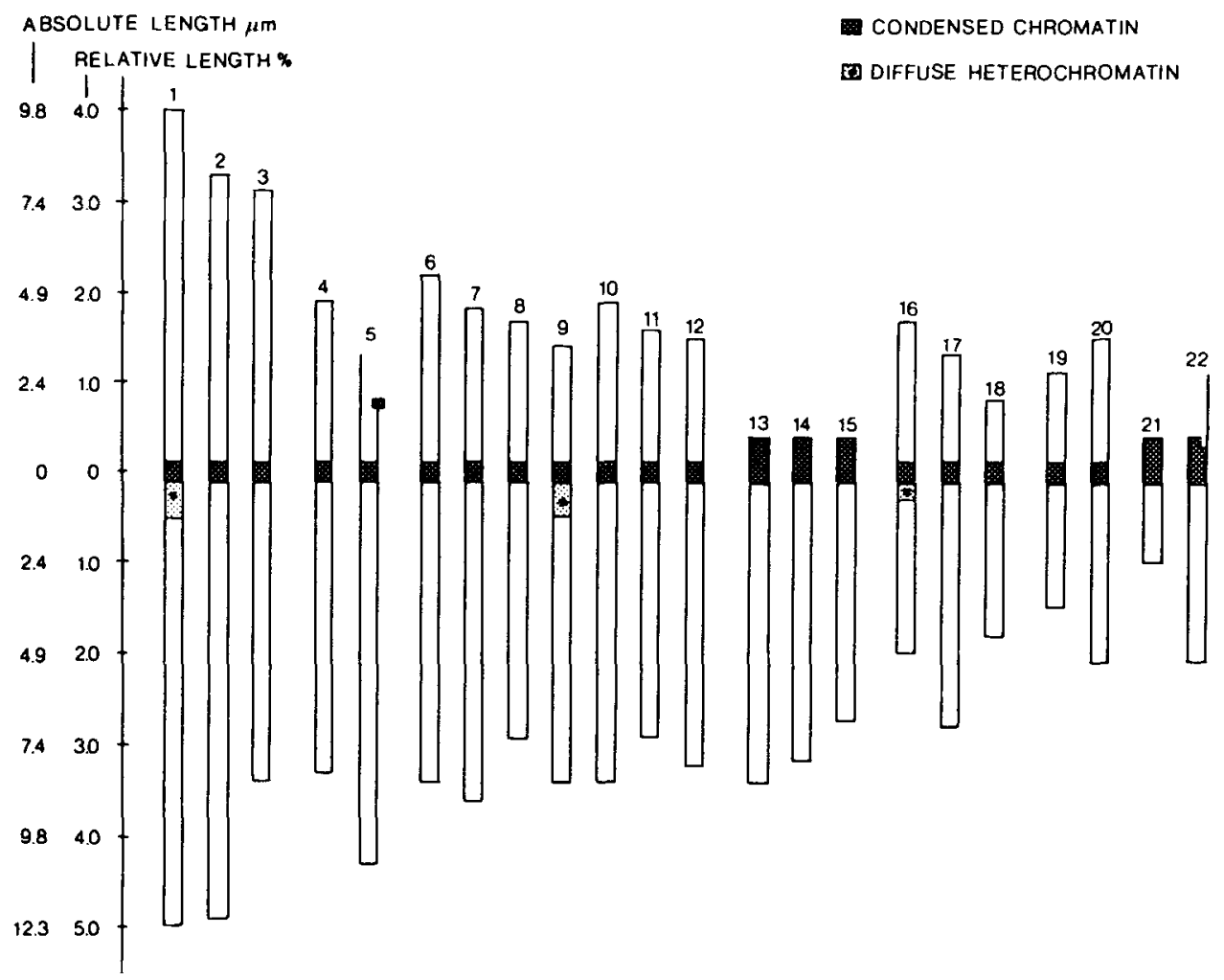

Figure 11. Idiogram showing the mean absolute and relative synaptonemal complex lengths of the 22 autosomal bivalents from four mid pachytene nuclei. The position of the centromeric heterochromatin is indicated by a dark hatching and the position of the diffuse heterochromatin (secondary constriction) of bivalents 1,9 and 16 by a lighter hatching. Bivalents 5 and 22 are drawn as heterozygous for a reciprocal translocation. 
The total length of the chromosomes in mitotic metaphase is $88 \mu \mathrm{m}$ (cf. Table 5 in 23) versus $244 \mu \mathrm{m}$ in mid pachytene. In the present case the segment in question with an estimated pachytene length of $200 \mathrm{~nm}$ would be less than $100 \mathrm{~nm}$ at mitotic metaphase and thus too small to be seen in the light microscope. In the latter, a large terminal chromosome segment can be identified and recognized, the so-called $\mathrm{T}$ band, which probably includes the telomere region. In this translocation $(5 p ; 22 p)$ the $T$ band of chromosome 5 is found (22) on the tip of the translocation chromosome 22 . The $T$ band of chromosome 22 on the other hand was not present at the tip of translocation chromosome 5 . This is explained by the electron microscopical finding which shows that only the telomere tip of this band consisting of condensed chromatin has been fused to the broken chromosome 5 .

The ultrastructure of the attached lateral component of the telomere in meiotic prophase nuclei has been discribed in a number of organisms (e.g., 8, 10, 11, 25, 27, 33). Although the ultrastructure of the meiotic telomere appears to differ in plants and animals, there is agreement that the telomere is a highly specialized chromosome region which plays a role in the primary recognition of homologous chromosomes at the nuclear envelope and their subsequent intimate pairing by formation of the synaptonemal complex. In Lillium and Bombyx it has been documented that the lateral component of the telomeres retains the terminal modification characteristic for the attachment sites even after their release from the nuclear envelope $(11,25)$.

In triploid Bombyx oocytes and in human spermatocytes, broken lateral components and fragments are occasionally found, in Bombyx as supernumerary fragments (26) and in human spermatocytes probably during the resolution of bivalent interlockings (27). The broken ends are in both cases always free in the nucleoplasm and without the terminal modification of the lateral component characteristic of attached and free telomeres.

These observations provide additional support to the conclusion that an intact and functionally active telomere is present on the translocation chromosome 5 of the present study.
Hsu (14) proposed that "interstitial telomeres" may be present along the chromosomes and upon breakage of the chromosome assume the function of a normal telomere. This idea, as argued by NIEBUHR (22) would require the presence of numerous interstitial telomeres in each chromosome since the break points in $355 \mathrm{p}$ individuals were distributed within the distal $60 \%$ of the short arm of chromosome 5 . Such a number and distribution of "interstitial telomeres" is considered unlikely.

The behaviour of broken chromosome ends during the breakage-fusion-bridge cycle in maize reported by MCCLINTOCK (18) excludes that a telomere is formed following chromosome breakage in the gametophyte and the endosperm. Whether the "healing" reported for the sporophyte implies that functional telomeres can be formed in certain developmental stages as modifications of the end of DNA double helices in order to prevent fusion of sister chromatids remains to be determined.

\subsection{Pairing of chromosomes 5 and 22}

The analysis of chromosome pairing in pachytene as a tool in the study of chromosome rearrangements is strictly dependent upon the specificity of chromosome pairing, i.e., whether synaptonemal complex formation occurs between homologous chromosome regions only. Most reports on chromosome pairing substantiate that pairing with synaptonemal complex formation is highly specific, i.e., that the presence of a synaptonemal complex signifies homologous pairing. Several studies have, however, revealed that this rule is not without exceptions. Taking inversion heterozygotes as one example, chromosome pairing may be either homologous with formation of an inversion loop or it may be nonhomologous, the synaptonemal complex passing unaffected through the inverted region $(6,8)$. Another example is the chromosome pairing in haploid barley, where up to $60 \%$ of the chromosome complement has been reported to pair nonhomologously with apparently normal synaptonemal complexes (9).

A solution to this dilemma was found through the analysis of triploid Bombyx oocytes 
(26) in which chromosome pairing during zygotene is strictly homologous resulting at early pachytene in homologously paired bivalents and trivalents in addition to univalents. Later in pachytene, trivalents are absent whereas many univalents are nonhomologously paired either by folding back on themselves or as nonhomologous associations of 2,3 , or 4 chromosomes. These observations were interpreted as being the result of a correction process whereby the trivalents formed during the homologous pairing phase in zygotene were first reorganized into bivalents and univalents through a dissolution and reassembly of the synaptonemal complex. Thereafter, in a second round of synaptonemal complex formation which does not require homology, nonhomologous assocations can form. It was proposed that this correction by dissolution and reassembly of the synaptonemal complex is only possible in the absence of crossing over as is the case in Bombyx females. This change in specificity of chromosome pairing and synaptonemal complex formation from early to late pachytene has also been indicated by preliminary data on synaptonemal complex formation in mice heterozygous for an inversion or a tandem duplication (19).

At early pachytene the close agreement between the length of the synaptonemal complex combining the normal chromosome 5 with translocation chromosome 22 and the differences in lateral component lengths of the normal and translocation chromosomes shows that the translocated segment is homologously paired throughout its entire length. The absence of pairing and synaptonemal complex formation between the $200 \mathrm{~nm}$ segment translocated from chromosome 22 to chromosome 5 and its homologous segment on chromosome 22 may be attributed to its small size in combination with mechanical restrictions of quadrivalent formation.

In contrast to the strictly homologous zygotene pairing in this translocation heterozygote, two examples of nonhomologous pairing with synaptonemal complex formation were revealed in one of the four mid pachytene nuclei (nucleus 29; pairing between the $\mathrm{X}$ chromosome and the normal chromosome 5 , and foldback pairing of the translocated segment on chromo- some 22). It is furthermore conceivable that the synaptonemal complex between translocation chromosome 5 and normal chromosome 22 in nucleus 28 is in part nonhomologous, as this short stretch of complex extends more than 200 $\mathrm{nm}(400 \mathrm{~nm})$ from the telomere of chromosome 5.

In all four mid pachytene nuclei, chromosomes 5 and 22 were intimately associated with the $\mathrm{XY}$ bivalent, while a similar association was absent at early pachytene (the quadrivalent and the $X Y$ bivalents were completely separated in all 8 nuclei). This nonspecific attraction between unpaired chromosome regions probably coincides with the second round of synaptonemal complex formation and may promote nonhomologous synaptonemal complex formation.

From the preceeding discussion it is evident that the first round of chromosome pairing during zygotene reveals a specific site to site matching of homologous chromosome regions at the zygotene-pachytene transition while the pairing pattern observed later in pachytene is the result of a modification of the specific zygotene pairing through an additional round of nonhomologous pairing. The analysis of chromosome pairing as an unquivocal test for homology of rearranged chromosome segments is consequently most meaningful at the zygotene-pachytene transition.

\subsection{Recombination nodules}

It has been proposed $(26,27)$ that crossing over in a given bivalent segment may prevent subsequent correction or modification of chromosome pairing in this chromosome or chromosome arm. Assuming that a recombination nodule at early pachytene signifies a crossing over event, then crossing over has occurred in the long translocated segment in at least 5 out of the 8 early pachytene nuclei (Table II). It is therefore conceivable that the preservation of a quadrivalent in 3 of the 4 mid pachytene nuclei is the result of crossing over within this translocated segment. A reorganization of the quadrivalent into two bivalents as observed in the fourth mid pachytene nucleus may be due to the absence of crossing over in the translocated segment. 
Pairing between the translocated $200 \mathrm{~nm}$ segment on translocation chromosome 5 and its homologous segment on normal chromosome 22 was, as previously mentioned, not observed in the 8 early pachytene nuclei. Had pairing occurred between these segments, the chance of a stabilization by crossing over would have been very low since recombination nodules and chiasmata generally appear to be absent in the short arms of the acrocentric bivalents $(27,15)$. The probability of an association of one nodule to a $200 \mathrm{~nm}$ segment of the short arm of bivalent 22 is $1.3 \%$. It is thus probable that the short stretch of complex between translocation chromosome 5 and normal chromosome 22 in nucleus 28 (Figure 10) was formed during the second, unspecific round of pairing.

The present study has demonstrated a significant increase in the number of recombination nodules in bivalents 5,9 , and 18 , and a small, nonsignificant decrease in bivalent 22. Effects of structural rearrangements on crossing over frequencies in regions of the involved chromosomes outside the rearrangments as well as in the remainder of the chromosome complement have been reported in many organisms (17). Although the present material is insufficient for unequivocal conclusions, it might be hypothesized that the alteration of nodule number encountered for bivalents 5 and 22 are somehow related to the mechanics of quadrivalent formation.

\section{ACKNOWLEDGEMENTS}

It is a great pleasure to thank dr. ERIK NIEBUHR, University Institute of Medical Genetics, Tagensvej 14, Copenhagen, Denmark, for his generous help in obtaining the testicular biopsy and for his encouragement during the course of the work. We also wish to thank Prof. D voN WETTSTEIN for valuable discussions and for his critical review of the manuscript. The outstanding technical assistance of JEAN SAGE, ANN-SOFI Steinholtz, Nina RASMUSSEN, Lena KongSRUD and INGE SOMMER is gratefully acknowledged. The work was supported by grant 202-76-1 BIO DK from the Commission of the European Communities.

\section{REFERENCES}

1. Bateman, A.J.: Simplification of palindromic telomere theory. Nature 253, 379 (1975)

2. Cavalier-Smith, T.: Palindromic base sequences and replication of eukaryote chromosome ends. Nature 250, 467-470 (1974)

3. DANCIS, B.M. \& G.P. HolmQuist: Fusion model of telomere replication and its implications for chromosome rearrangements. In: $A$. de la Chapelle \& M. Sorsa, eds., Chromosomes Today 6, 95-104 (1977)

4. De la Chapelle, A. \& J. Schróder: Apparently non-reciprocal balanced human $(3 q-; X q+)$ translocation: late replication of structurally normal X. In: J. Wahrmann \& K.R. Lewis, eds., Chromosomes Today 4, 261-265 (1973)

5. Demerec, M. \& M.E. Hoover: Three related $X$ chromosome deficiencies in Drosophila differing in extent of deleted material and in viability. $J$. Heredity 27, 206-212(1936)

6. Fletcher, H.L. \& G.M. HewITT: Non-homologous synaptonemal complex formation in a heteromorphic bivalent in Keyacris scurra (Morabinae, Orthoptera). Chromosoma (Berl.) 65, 271-281 (1978)

7. FranCKe, U.: Quinacrine mustard fluorescence of human chromosomes: Characterization of unusual translocations. Amer. J. Hum. Genet. 24, 189-213 (1972)

8. GiLlies, C.B.: Ultrastructural analysis of maize pachytene karyotypes by three dimensional reconstruction of the synaptonemal complexes. Chromosoma (Berl.) 43, 145-176 (1973)

9. Gillies, C.B.: The nature and extent of synaptonemal complex formation in haploid barley. Chromosoma (Berl.) 48, 441-453 (1974)

10. Gillies, C.B.: Synaptonemal complex and chromosome structure. Ann. Rev. Genet. 9, 91-111 (1975)

11. HoLm, P.B.: Three-dimensional reconstruction of chromosome pairing during the zygotene stage of meiosis in Lilium longiflorum (Thunb.). Carlsberg Res. Commun. 42, 103-151 (1977)

12. Holm, P.B. \& S.W. RaSmusSEN: Human meiosis I. The human pachytene karyotype analyzed by three dimensional reconstruction of the synaptonemal complex. Carlsberg Res. Commun. 42, 283-323 (1977)

13. Holm, P.B. \& S.W. Rasmussen: Three-dimensional reconstructions of meiotic chromosomes in human spermatocytes. In: A. de la Chapelle \& M. Sorsa, eds., Chromosomes Today 6, 83-93 (1977)

14. Hsu, T.C.: Longitudinal differentiation of chro- 
mosomes and the possibility of interstitial telomeres. Exptl. Cell. Res. Suppl. 9, 73-83 (1963)

15. Hulten, M.: Chiasma distribution at diakinesis in the normal human male. Hereditas $76,55-78$ (1974)

16. LIMA-DE-FARIA, A.\& P. SARVELlA: The organization of telomeres in species of Solanum, Salvia, Scilla, Secale, Agapanthus and Ornithogalum. Hereditas 44, 337-346 (1958)

17. LuCCHESI, J.C. \& D.T. SuZUKI: The interchromosomal control of recombination. Ann. Rev. Genet. 2, 53-86 (1968)

18. MCC.INTOCK, B.: The stability of broken ends of chromosomes in Zea mays. Genetics 26, 243-282 (1941)

19. Moses, M.J.: Microspreading and the synaptonemal complex in cytogenetic studies. In: A. de la Chapelle \& M. Sorsa, eds., Chromosomes Today 6, 71-82 (1977)

20. Muller, H.J.: Further studies on the nature and causes of gene mutations. Proc. VI Intern. Cong. Genet. 1, 213-255 (1932)

21. Muller, H.J. \& I.H. Herskowitz: Concerning the healing of chromosome ends produced by breakage in Drosophila melanogaster. Amer. Naturalist 88, 177-208 (1954)

22. Niebuhr, E.: Cytologic observations in 35 individuals with a $5 \mathrm{p}$ - karyotype. Hum. Genet. 42 , 143-156 (1978)

23. PARIS CONFERENCE (1971): Standardization in human cytogenetics. Cytogenetics 11, 313-362 (1972)

24. Perkins, D.D. \& E.G. Barry: The cytogenetics of Neurospora. Advances in Genetics 19, 133-285 (1977)

25. RASMUSSEN, $S . W$ : The meiotic prophase in Bombyx mori females analyzed by three-dimensional reconstructions of synaptonemal complexes. Chromosoma (Berl.) 54, 245-293 (1976)

26. Rasmussen, S.W.: Chromosome pairing in triploid females of Bombyx mori analyzed by three dimensional reconstructions of synaptonemal complexes. Carlsberg Res. Commun. 42, 163-197 (1977)

27. Rasmussen, S.W. \& P.B. Holm: Human meiosis II. Chromosome pairing and recombination nodules in human spermatocytes. Carisberg Res. Commun. 43, (1978)

28. RoBerts, P.A.: In support of the telomere concept. Genetics 80, 135-142 (1975)

29. Rubin, G.M.: Isolation of a telomeric DNA sequence from Drosophila melanogaster. Cold Spring Harb. Symp. quant. Biol. 42, 1041-1046 (1978)

30. SHaw, M.W.: Annotation: Human chromosome abnormalities revisited. Amer. J. Hum. Genet. 24, 227-228 (1972)

31. Sutton, E.: Terminal deficiencies in the $X$ chromosome of Drosophila melanogaster. Genetics 25, 628-635 (1940)

32. WATSON, J.D.: Origin of concatemeric T7 DNA. Nature 239, 197-201 (1972)

33. WestergaARD, M. \& D. von Wettstein: The synaptinemal complex. Ann. Rev. Genet. 6, $71-110(1972)$ 